Less familiar, perhaps, is the stridulation of certain spiders. Although not so noticeable as the "song" of the cricket, it can, nonetheless, be picked up by the human ear, as Professor H. E. Hinton discovered when he was collecting spiders in 1969 in a Mexican garden. While taking a spiny orb-weaver (Micrathena gracilis) from its net, he heard a low pitched buzz which was audible at a distance of about 2 feet. Later, Hinton and his colleague, R. S. Wilson, found that this species possesses a well developed stridulatory organ with a file on the cover of the lung book (the respiratory organ) (J. Zool., 162, 481; 1970). The scraper consists of three or four stout setae which project dorsally from a small area near the base of the hind femur (see figure). The setae are curved posteriorly and are fairly flexible, and the buzz or hiss is produced when the points of the setae move across the file. The lung book, Hinton and Wilson suggest, may act as a resonance cavity that amplifies the sound.

Seven different types of stridulatory organ have now been distinguished in spiders according to the positions of the files and scrapers. Hinton and Wilson's description of a stridulatory organ in Micrathena is the first record of the organ in the family Argiopidae although a similar type of organscrapers on the hind legs and files on the covers of the lung books-has been found in a few genera of Erigonidae.

Hinton and Wilson have so far found stridulatory organs in only two species of Micrathena. It is interesting that there are specific differences in the spacing of the ridges on the files; in $M$. gracilis the distance from the middle of one ridge to the middle of the next is $8.3-12.0 \mu \mathrm{m}$, with most ridges spaced about $11 \mu \mathrm{m}$ apart; in $M$. schreibersi, on the other hand, the ridges are much closer together (5.0-6.6 $\mu \mathrm{m})$ and in most areas the spacing is $5.5 \mu \mathrm{m}$. In this species therefore the ridges are close enough together to give bright diffraction spectra at incidences near grazing.

The stridulatory organs in spiders could have several possible functions. Courtship is an obvious one but Hinton and Wilson suggest that in Micrathena the sound is more likely to function as a warning signal because it is produced when the spider is touched or otherwise disturbed.

\section{DRUGS}

\section{Defecting Dependence}

from a Correspondent

THE meeting on addictive drugs held at the Institute of Psychiatry, University of London, on January 22, 1971, was sponsored by two different groups of the Biochemical Society (Neurochemistry and Pharmacological Biochemistry) and

turned out to have two quite different themes. One theme was the role of body fluid analysis in the management of drug abuse. The other concerned mechanisms of drug dependence in rodents, particularly the possible relationship between morphine dependence and brain 5-hydroxytryptamine. Each theme is important to the problem of drug addiction in quite a different waythe one in therapy, the other in understanding basic dependence mechanismsand each was dealt with thoroughly; but the many other aspects of the vast subject covered by the title were omitted.

On the analytical theme, Dr P. H. Connell (Maudsley Hospital, London) pointed out that, whereas most psychiatric patients gave honest answers to questions, compulsive drug takers could not be relied on to do so. The resulting difficulties are greater with younger addicts, who often practise multiple drug abuse. Objective tests are therefore needed to tell the doctor whether the patient is taking (1) unprescribed drugs and, if so, what drugs and how much of each, and (2) the correct doses of drugs prescribed. Without this information, mistakes can be made; for example, a sporadic drug taker may be turned into a regular taker or a recipient may sell the prescribed drug instead of taking it. Because blood is difficult to obtain from "main liners" and blood levels of drugs

\title{
Identification of Cells producing Interferon
}

INOCULATIONS of viruses, some other microorganisms or double stranded polyribonucleotides into animals are followed by the appearance of interferon in the circulating plasma. The interferon can be titrated accurately by its capacity to prevent the replication of viruses in cultured cells.

Because interferon may be involved in host protection against infection, it is of interest to identify the cells by which it is produced. Jacqueline and Edouard de Maeyer of the Institut du Radium, Paris, report in next Wednesday's Nature New Biology that interferon induction by Newcastle disease virus (NDV) is radiosensitive and its production in mice is depressed by more than 90 per cent after $X$-radiation of $250 \mathrm{r}$. The species specificity of interferon enabled the Maeyers to establish that in xenogeneic radiation chimaeras the circulating interferon induced by NDV is of donor origin, from which it can be concluded that the cells synthesizing interferon are derived from blood-forming stem cells.

Both granulocytes and lymphocytes are radiosensitive progeny of such stem cells, and anti-lymphocytic serum (ALS) was used to identify the cells producing interferon. Interferon induction by NDV was found to be markedly depressed by are difficult to interpret, reliance is usually placed on urine analysis.

Dr Monamy Buckell (Bethlem Royal and Maudsley Hospitals, London) outlined the difficulties of detecting and estimating drugs in urine. Apart from the heavy load of samples, there was the lack of good methods of estimating cannabis or LSD and the unreliable course of excretion of a drug or its metabolites. Professor A. H. Beckett (Chelsea College, University of London) emphasized the importance of urinary $p \mathrm{H}$ in regulating the pattern of excretion, illustrating this with the effect of acidity of urine on excretion of amphetamines and their metabolites and of methadone.

On the rodent theme, Dr H. O. J. Collier and $\mathrm{Mr} \mathrm{C}$. Schneider (Miles Laboratories Ltd, Stoke Poges) said that psychic and physical dependence in the rat and physical dependence in the mouse on opiates had only been clearly recognized in recent years, partly because opiate antagonists became available to accentuate withdrawal effects. Because physical dependence develops quickly in these species and because its intensity can be quantitated by counting jumps and other obvious withdrawal effects, the rat and mouse offered a rapid means of obtaining experimental data on physical dependence. These authors described the failure in their laboratory of attempts to confirm the conclusions of Shen, Loh and Way injections of ALS, which selectively decreased peripheral lymphocyte counts and may also have affected lymphocyte function. This suggests that myxoviruses such as NDV elicit interferon production chiefly by lymphocytes. Indeed, the release of interferon from lymphocytes exposed to viruses, specific antigens such as tuberculin and non-specific mitogens such as phytohaemagglutinin has attracted attention during the past few yearsinterferon production may be one of the derepressed functions in the lymphocyte exposed to viruses or transformed.

It would be premature, however, to conclude that the lymphocyte is the only cell from which circulating interferon can be derived. Interferon induction by encephalomyocarditis virus, vaccinia virus or polyriboinosinic:polyribocytidylic acid is relatively resistant to radiation or ALS; interferon may be synthesized by macrophages or other cells in these cases. Interferon induction by arboviruses or vesicular stomatitis virus shows intermediate responses to radiation or ALS, less than that on interferon induction by myxoviruses but greater than that on interferon induction by encephalomyocarditis virus. Perhaps some, but not all, of the circulating interferon is synthesized by lymphocytes in these intermediate cases. 